\title{
Produção científica brasileira de enfermagem sobre erros de medicação
}

\author{
Francisco Gilberto Fernandes Pereira*, Rebeca Barros da Silva**, Márcia Barroso Camilo de Ataíde, D.Sc.***, \\ Joselany Áfio Caetano, D.Sc. ${ }^{* * *}$
}

*Enfermeiro, especialista em farmacologia, Docente do centro de saúde do Centro Universitário Estácio do Ceará, **Enfermeira da gerência de risco hospitalar do Hospital Geral de Fortaleza, Especialista em Enfermagem do Trabalho, ***Enfermeira da Secretaria de Saúde do Estado do Ceará, Docente do Centro de Ciências da Saúde da Universidade de Fortaleza, ${ }^{* * *}$ Docente do curso de enfermagem da Universidade Federal do Ceará

\section{Resumo}

O objetivo foi analisar o conhecimento científico produzido por enfermeiros em relação aos erros de medicação. Realizou-se uma revisão integrativa nas bases de dados Pubmed, Scielo, BDEnf e Lilacs. A amostra contou com 31 artigos publicados entre 2000 e 2010. Os resultados evidenciaram que o periódico Acta Paulista de Enfermagem apresentou a maior quantidade de publicaçôes, $48 \%$ dos autores são enfermeiros doutores que trabalham apenas na docência. Os erros mais comumente referenciados foram os de hora e dose, destacando-se como principais fatores relacionais o profissional (problemas de comunicaçáo, falta de treinamento, elevada carga horária de trabalho) ou o sistema (ausência de dose unitária, dificuldade de interpretação da prescrição), e recomendações foram sugeridas a curto, médio e longo prazo, com a internalização de uma cultura de segurança do paciente. Conclui-se que há uma crescente publicação de enfermagem na área de erros de medicaçáo, e sugerem-se estudos de avaliaçáo de impacto.

Palavras-chave: erros de medicação, segurança do paciente, Enfermagem.

\section{Abstract}

\section{Scientific production in Brazilian nursing about medication errors}

The aim of this study was to analyze the scientific knowledge produced by nurses on medication errors. An integrative review was developed in Pubmed, Scielo, BDEnf and Lilacs databases. The sample included 31 papers, published between 2000 and 2010. The results evidenced that the largest number of publications came from the journal Acta Paulista de Enfermagem, and that $48 \%$ of the authors are nursing Ph.D.'s who are only active in teaching. The most common medication errors were time and dose errors, and the professional (communication problems, lack of training, high hour load) or system (absence of unit dose, prescription interpretation difficulty) were highlighted as the main related factors. Recommendations were suggested in the short, medium and long term, involving the internalization of a patient safety culture. In conclusion, the number of nursing publications on medication errors is increasing, and impact assessment studies are suggested.

Key-words: medication errors, patient safety, Nursing. 


\section{Resumen}

\section{Producción científica brasileña sobre errores de medicación}

La finalidad fue analizar el conocimiento científico producido por enfermeros sobre errores de medicación. Fue efectuada una revisión integradora en Pubmed, Scielo, BDEnf y Lilacs. La muestra abarcó un total de 31 artículos, publicados entre 2000 y 2010. Los resultados evidenciaron que Acta Paulista de Enfermagem divulgó la mayor cantidad de publicaciones. El 48\% de los autores son enfermeros doctores activos sólo en la docencia. Los errores más comúnmente referidos fueron los de hora y dosis, destacándose como principales factores relacionados el profesional (problemas de comunicación, falta de capacitación, elevada carga horaria de trabajo) o el sistema (ausencia de dosis unitaria, dificultad de interpretación de la prescripción), y recomendaciones fueron sugeridas a corto, medio y largo plazo, con la internalización de una cultura de seguridad del paciente. Se concluye que existe una creciente publicación de enfermería en el área de errores de medicación, y se sugieren estudios de evaluación de impacto.

Palabras-clave: errores de medicación, seguridad del paciente, enfermaría.

\section{Introdução}

Erro de medicação é definido, consoante à taxonomia de segurança do paciente, como aquele evento que se trata de um erro evitável, provocado devido ao uso inadequado de medicamentos, e que pode acontecer em qualquer etapa do sistema de medicação, seja relacionado ao produto, ao profissional ou ao procedimento [1].

Com relação ao sistema de medicação, estudo realizado no Brasil já alertava que 39\% dos erros ocorrem durante a prescrição, $12 \%$ na transcrição, $11 \%$ na dispensação e 38\% durante a administração. Enfatizou-se ainda, que Enfermeiros e farmacêuticos interceptam $86 \%$ dos erros de medicação nas etapas de prescrição, transcrição e dispensação, enquanto apenas 2\% são interceptados pelos pacientes [2]. Porém, um erro em qualquer etapa no processo de medicação pode acarretar danos irreparáveis ao paciente, inclusive óbito, como descrevem vários estudos na literatura mundial $[3,4]$. Assim, na primeira década do século XXI, foi criada pela Organizaçáo Mundial de Saúde (OMS) uma Aliança Mundial para a Segurança do Paciente [5] com o objetivo de socializar os principais problemas que afetam a segurança na assistência ao paciente que necessita dos serviços de saúde, e possíveis soluçóes para esses entraves.

Dentre as principais metas idealizadas pela Aliança, está a segurança na utilização das medicaçóes, tópico que mereceu destaque mais acentuado após a divulgação de um relatório de erros no sistema de saúde nos Estados Unidos em 2000, que categorizou o erro de medicação como responsável direto pela morte de 7391 pacientes. No Brasil, embora alguns estudos tenham destacado a presença desses erros, não é possível ainda inferir o seu real dimensionamento $[3,6]$.

A administração de medicamento é uma das maiores responsabilidades da equipe de enfermagem. Tal condição determina que essa prática seja exercida de modo adequado e seguro. Embora haja amparo legal e formação curricular para a realização desta atividade, verifica-se que, muitas vezes, há despreparo do profissional para fazê-la, sua execução pode ocorrer de maneira automática e desatenciosa, desconsiderando-se o impacto que um erro nesse processo pode desencadear [7].

A operacionalizaçáo do manuseio, preparo e administração de medicamentos expóe os profissionais de enfermagem a várias situaçóes de passividade de erros, sendo considerados os auxiliares e técnicos de enfermagem como os mais vulneráveis $[8,9]$.

Considerando essa contextualização acerca da segurança do paciente na administração de medicamentos, e compreendendo que a equipe de enfermagem realiza este tipo de procedimento de forma repetitiva, o estudo tem como objetivo analisar o conhecimento científico produzido por enfermeiros sobre os erros de medicação.

A realização de uma revisão integrativa com essa abordagem será importante para apresentar a síntese das produçóes, suas lacunas, oferecer um suporte na melhoria da tomada de decisóes, bem como criação de novas estratégias na redução do erro relacionado à medicação pelo profissional de enfer- 
magem. Quanto mais amplo for o conhecimento e a divulgação dos fatores causadores de erros de medicação, maiores as possibilidades de prevenção deste evento.

\section{Material e métodos}

Para o alcance do objetivo do estudo, optou-se pelo método da revisão integrativa, por oportunizar uma sumarização das pesquisas já concluídas e obter inferências a partir de uma temática de interesse. Para a efetivação deste estudo foi necessário operacionalizar a revisão seguindo cronologicamente as seguintes etapas: seleção da questão temática, estabelecimento dos critérios para a seleção dos artigos, representaçáo das características da pesquisa original, análise dos dados, interpretaçáo dos resultados e apresentação da revisão [10].

Considerando a problemática da pesquisa e para guiar a presente revisão, formulou-se a seguinte questão norteadora: qual o conhecimento científico produzido pela enfermagem no Brasil sobre erro de medicaçáo?

O levantamento bibliográfico foi realizado pela internet, nas bases de dados Scielo, PubMed, BDEnf e Lilacs durante os meses de janeiro a março de 2011, utilizando os descritores: erros de medicaçáo (medication errors) and enfermagem (nursing), de acordo com os Descritores em Ciências da Saúde (DeCS).

Os critérios utilizados para seleção da amostra foram: artigos publicados eletronicamente na íntegra, nos idiomas inglês, português ou espanhol, que abordassem a temática de administração de medicamentos na área de enfermagem, com autoria de pelo menos um enfermeiro, pesquisa realizada no Brasil, no período de 2000 a 2010, justificando-se por ter sido a década de divulgaçáo do relatório de erros dos EUA e criação da Aliança Mundial para Segurança do Paciente. Foram excluídos do estudo: editoriais, cartas ao leitor e estudo que não abordassem a temática relevante ao alcance do objetivo da revisão.

Na busca inicial foram encontrados 133 artigos, sendo 19 na base BDENF, 56 na Lilacs, 18 na Pubmed e 40 na Scielo. Após leitura dos resumos disponíveis e orientando-se pela questão norteadora, foram excluídos 67, restando ao final 66 artigos, dos quais $11 \mathrm{da}$ BDEnf, $22 \mathrm{da}$ Lilacs, $11 \mathrm{da}$ Pubmed e 22 da Scielo, que após análise de duplicidade nas diferentes bases pesquisadas restaram 31 artigos.
Para a classificação do material foi realizada leitura analítica dos artigos na íntegra e preenchido um instrumento que permitiu obter informaçóes sobre autores (formação técnica, acadêmica e campo de atuação) e sobre os artigos (identificação do artigo, local de origem e ano da publicação, nome do periódico, objetivo do estudo, metodologia utilizada e recomendaçóes para a prática de enfermagem).

Os resultados estáo apresentados de forma descritiva. A análise se processou de forma descritiva com apoio na literatura concernente às tendências e prioridades da pesquisa em enfermagem na especificidade da administração de medicamentos e segurança do paciente, bem como de reflexóes e críticas dos autores, enfocando os resultados e recomendaçóes para a prática assistencial.

\section{Resultados e discussão}

Dos 31 artigos selecionados com a temática de erros de medicação na área de enfermagem, constatou-se que o periódico Acta Paulista de Enfermagem foi o que contabilizou maior volume de publicação com oito artigos. Os demais periódicos que divulgaram estudos relacionados ao tema em estudo foram: Revista Latino Americana de Enfermagem (7), Revista Brasileira de Enfermagem (7), Revista da Escola de Enfermagem da USP (2), Revista de Enfermagem da UFPE (2), Arquivos Ciência e Saúde UNIPAR (1), Revista Brasileira de Terapia Intensiva (2), Medicina (1) e Revista de Enfermagem da UERJ (1).

Este aspecto implica dizer que ainda há um monopólio das regióes sul e sudeste na formação crítica e na disseminação do conhecimento científico, e que os periódicos de outras regióes, principalmente o nordeste, necessitam ampliar a visão sobre o tema erros de medicação e criar espaços de divulgação, já que esta é uma temática tão recorrente no cotidiano profissional e tão atual e necessária.

Acerca dos autores é possível inferir que há uma predominância do número de enfermeiros com doutorado (12), sendo que destes, a maioria (11) atua como docente em Universidades. Identificaram-se dez enfermeiros com mestrado, prevalecendo também como área de atuação a docência. Por outro lado, os enfermeiros com titulação de especialista atuam somente na assistência.

Com relação ao ano, nota-se que 2010 foi o período em que há registros do maior número de publicaçôes, totalizando sete. Esse número maior de 
artigos em 2010 corrobora as ideias pregoadas pela Aliança Mundial para Segurança do Paciente, que tinha como metas permeabilizar o conhecimento científico e fomentar pesquisas na área de medicamentos e segurança do paciente [5]. Não foram encontrados artigos nos anos de 2001 e 2004.

É interessante que os enfermeiros envolvidos na pesquisa em geral, estejam envolvidos com atividades assistenciais, pois, a prática cotidiana gera questionamentos e permite experimentar ideias que são inexistentes nos centros universitários [11].

Dentro desse universo de autores, dez dos artigos analisados foram escritos por enfermeiros em associação com farmacêuticos. Esse dado aponta para uma nova realidade que se vislumbra no cenário da saúde que é a consolidaçáo do trabalho em equipe interdisciplinar, e que também é preconizada para a diminuição de erros com medicamentos [12], pois proporciona a visão ampla de todo o sistema de medicação, suas possíveis falhas, e visóes amplas de correção das mesmas. Entretanto, seria interessante que outros profissionais se integrassem a esse time, como médicos, odontólogos, enfim, todos aqueles que estão envolvidos na segurança do paciente.

As ideias dos diversos profissionais de saúde e suas observaçóes concernentes aos erros de medicação, certamente resultaria em novas inferências, novos referenciais teóricos e surgimento de estratégias interdisciplinares para assegurar acertos de medicação.

Baseado na caracterização metodológica [13] dos estudos, observou-se que houve uma profunda empatia por pesquisas exploratório-descritivas, seguidas de revisão bibliográfica. A escolha por métodos experimentais ou quase-experimentais apareceu timidamente nesta revisão, apesar de ser um tipo de investigação totalmente coerente com a temática de erros de medicação. Acredita-se, no entanto, que a pouca utilização desse método deveu-se a limitaçóes financeiras ou a inexperiência da enfermagem brasileira em realizar estudos dessa categoria metodológica.

Tabela I - Apresentação da síntese de artigos incluídos na revisão integrativa.

\begin{tabular}{|c|c|c|c|c|c|}
\hline Autores & Título do artigo & Ano & Local do estudo & Periódico & $\begin{array}{c}\text { Base de } \\
\text { dados }\end{array}$ \\
\hline $\begin{array}{l}\text { Carvalho VT et al. } \\
{[14]}\end{array}$ & $\begin{array}{l}\text { Erros na medicação: análise das situ- } \\
\text { ações relatadas pelos profissionais de } \\
\text { enfermagem. }\end{array}$ & 2000 & São Paulo & Medicina & Lilacs \\
\hline $\begin{array}{l}\text { Carvalho VT et al. } \\
\text { [15] }\end{array}$ & $\begin{array}{l}\text { Análise dos comportamentos dos } \\
\text { profissionais de enfermagem frente } \\
\text { aos erros na administração de medi- } \\
\text { camentos. }\end{array}$ & 2002 & São Paulo & $\begin{array}{l}\text { Acta Paul } \\
\text { Enferm }\end{array}$ & $\begin{array}{l}\text { Lilacs } \\
\text { BDEnf }\end{array}$ \\
\hline $\begin{array}{l}\text { Padilha KG et al. } \\
\text { [16] }\end{array}$ & $\begin{array}{l}\text { Ocorrências iatrogênicas com medica- } \\
\text { ção em Unidade de Terapia Intensiva: } \\
\text { condutas adotadas e sentimentos } \\
\text { expressos pelos enfermeiros. }\end{array}$ & 2002 & São Paulo & $\begin{array}{l}\text { Rev Esc En- } \\
\text { ferm USP }\end{array}$ & $\begin{array}{l}\text { Lilacs } \\
\text { Pubmed } \\
\text { Scielo }\end{array}$ \\
\hline $\begin{array}{l}\text { Carvalho VT et al. } \\
{[17]}\end{array}$ & $\begin{array}{l}\text { Erros na medicação e consequências } \\
\text { para profissionais de enfermagem e } \\
\text { clientes: um estudo exploratório. }\end{array}$ & 2002 & São Paulo & $\begin{array}{l}\text { Rev Latinoam } \\
\text { Enferm }\end{array}$ & Pubmed \\
\hline Helena B et al. [18] & $\begin{array}{l}\text { Erros de medicação - causas e fatores } \\
\text { desencadeantes sob a ótica da equipe } \\
\text { de enfermagem. }\end{array}$ & 2003 & São Paulo & $\begin{array}{l}\text { Acta Paul } \\
\text { Enferm }\end{array}$ & Lilacs \\
\hline $\begin{array}{l}\text { Camargo MNV et } \\
\text { al. [19] }\end{array}$ & $\begin{array}{l}\text { Ocorrências iatrogênicas com medica- } \\
\text { ção em unidades de terapia intensiva. }\end{array}$ & 2003 & São Paulo & $\begin{array}{l}\text { Acta Paul } \\
\text { Enferm }\end{array}$ & Lilacs \\
\hline $\begin{array}{l}\text { Cassiani SHB et al. } \\
{[20]}\end{array}$ & $\begin{array}{l}\text { A prescrição médica eletrônica em } \\
\text { um hospital universitário: falhas de } \\
\text { redação e opiniões de usuários. }\end{array}$ & 2003 & São Paulo & $\begin{array}{l}\text { Rev Esc En- } \\
\text { ferm USP }\end{array}$ & $\begin{array}{l}\text { Lilacs } \\
\text { Pubmed } \\
\text { Scielo } \\
\end{array}$ \\
\hline Santos AE et al. [21] & $\begin{array}{l}\text { Eventos adversos com medicação em } \\
\text { serviços de emergência: condutas } \\
\text { profissionais e sentimentos vivenciados } \\
\text { por enfermeiros. }\end{array}$ & 2005 & São Paulo & $\begin{array}{l}\text { Rev Bras } \\
\text { Enferm }\end{array}$ & $\begin{array}{l}\text { Lilacs } \\
\text { Pubmed } \\
\text { Scielo } \\
\text { BDEnf } \\
\end{array}$ \\
\hline
\end{tabular}




\begin{tabular}{|c|c|c|c|c|c|}
\hline Autores & Título do artigo & Ano & Local do estudo & Periódico & $\begin{array}{c}\text { Base de } \\
\text { dados }\end{array}$ \\
\hline Melo LR et al. [22] & $\begin{array}{l}\text { Erros de medicação em pediatria: } \\
\text { análise da documentação de enferma- } \\
\text { gem no prontuário do paciente. }\end{array}$ & 2005 & São Paulo & $\begin{array}{l}\text { Rev Bras } \\
\text { Enferm }\end{array}$ & $\begin{array}{l}\text { Lilacs } \\
\text { Pubmed } \\
\text { Scielo } \\
\end{array}$ \\
\hline Oliveira RC [23] & $\begin{array}{l}\text { Estratégias para prevenção de erros } \\
\text { de medicação no setor de emergên- } \\
\text { cia. }\end{array}$ & 2005 & Recife & $\begin{array}{l}\text { Rev Bras } \\
\text { Enferm }\end{array}$ & $\begin{array}{l}\text { Lilacs } \\
\text { Pubmed } \\
\text { Scielo } \\
\text { BDEnf } \\
\end{array}$ \\
\hline $\begin{array}{l}\text { Cassiani SHB et al. } \\
{[24]}\end{array}$ & $\begin{array}{l}\text { O sistema de medicação nos hospi- } \\
\text { tais e sua avaliação por um grupo de } \\
\text { profissionais. }\end{array}$ & 2005 & São Paulo & $\begin{array}{l}\text { Rev Esc En- } \\
\text { ferm USP }\end{array}$ & $\begin{array}{l}\text { BDEnf } \\
\text { Scielo }\end{array}$ \\
\hline $\begin{array}{l}\text { Toffoletto MC et al. } \\
\text { [25] }\end{array}$ & $\begin{array}{l}\text { Conseqüências de erros de medicação } \\
\text { em unidades de terapia intensiva e } \\
\text { semi-intensiva. }\end{array}$ & 2006 & São Paulo & $\begin{array}{l}\text { Rev Esc En- } \\
\text { ferm USP }\end{array}$ & $\begin{array}{l}\text { Lilacs } \\
\text { Pubmed } \\
\text { Scielo } \\
\text { BDEnf } \\
\end{array}$ \\
\hline $\begin{array}{l}\text { Bohomol E et al. } \\
\text { [26] }\end{array}$ & $\begin{array}{l}\text { Perceptions about medication errors: } \\
\text { analysis of answers by the nursing } \\
\text { team. }\end{array}$ & 2006 & São Paulo & $\begin{array}{l}\text { Rev Latinoam } \\
\text { Enferm }\end{array}$ & $\begin{array}{l}\text { Lilacs } \\
\text { BDEnf }\end{array}$ \\
\hline Miasso Al et al. [6] & $\begin{array}{l}\text { O processo de preparo e administra- } \\
\text { ção de medicamentos: identificação } \\
\text { de problemas para propor melhorias } \\
\text { e prevenir erros de medicação. }\end{array}$ & 2006 & $\begin{array}{l}\text { Recife, São } \\
\text { Paulo, Goiânia } \\
\text { e Ribeirão Preto }\end{array}$ & $\begin{array}{l}\text { Rev Latinoam } \\
\text { Enferm }\end{array}$ & $\begin{array}{l}\text { Lilacs } \\
\text { Pubmed } \\
\text { Scielo }\end{array}$ \\
\hline $\begin{array}{l}\text { Yamanaka Tl et al. } \\
\text { [7] }\end{array}$ & $\begin{array}{l}\text { Redesenho de atividades da enfer- } \\
\text { magem para redução de erros de } \\
\text { medicação em pediatria. }\end{array}$ & 2007 & São Paulo & $\begin{array}{l}\text { Rev Bras } \\
\text { Enferm }\end{array}$ & $\begin{array}{l}\text { Lilacs } \\
\text { Pubmed } \\
\text { Scielo }\end{array}$ \\
\hline $\begin{array}{l}\text { Santos JO et al. } \\
\text { [27] }\end{array}$ & $\begin{array}{l}\text { Sentimentos de profissionais de enfer- } \\
\text { magem após a ocorrência de erros de } \\
\text { medicação. }\end{array}$ & 2007 & Goiânia & $\begin{array}{l}\text { Acta Paul } \\
\text { Enferm }\end{array}$ & $\begin{array}{l}\text { Lilacs } \\
\text { Scielo } \\
\text { BDEnf } \\
\end{array}$ \\
\hline $\begin{array}{l}\text { Bohomol E et al. } \\
\text { [28] }\end{array}$ & $\begin{array}{l}\text { Erro de medicação: importância da } \\
\text { notificação no gerenciamento da } \\
\text { segurança do paciente. }\end{array}$ & 2007 & São Paulo & $\begin{array}{l}\text { Rev Bras } \\
\text { Enferm }\end{array}$ & $\begin{array}{l}\text { Lilacs } \\
\text { Pubmed } \\
\text { Scielo } \\
\text { BDEnf } \\
\end{array}$ \\
\hline Silva BK et al. [8] & $\begin{array}{l}\text { Erros de medicação: condutas e pro- } \\
\text { postas de prevenção na perspectiva } \\
\text { da equipe de enfermagem. }\end{array}$ & 2007 & São Paulo & $\begin{array}{l}\text { Rev Eletrônica } \\
\text { Enferm }\end{array}$ & $\begin{array}{l}\text { Lilacs } \\
\text { BDEnf }\end{array}$ \\
\hline Silva DO et al. [29] & $\begin{array}{l}\text { Medication preparation and admi- } \\
\text { nistration: analysis of inquiries and } \\
\text { information by the nursing team. }\end{array}$ & 2007 & São Paulo & $\begin{array}{l}\text { Rev Latinoam } \\
\text { Enferm }\end{array}$ & $\begin{array}{l}\text { Lilacs } \\
\text { Scielo }\end{array}$ \\
\hline Silva AEBC [30] & $\begin{array}{l}\text { Problemas na comunicação: uma pos- } \\
\text { sível causa de erros de medicação. }\end{array}$ & 2007 & Goiás & $\begin{array}{l}\text { Acta Paul } \\
\text { Enferm } \\
\end{array}$ & $\begin{array}{l}\text { Lilacs } \\
\text { Scielo }\end{array}$ \\
\hline $\begin{array}{l}\text { Freitas DF et al. } \\
\text { [31] }\end{array}$ & $\begin{array}{l}\text { Avaliação dos fatores de risco relacio- } \\
\text { nados às falhas durante a administra- } \\
\text { ção de medicamentos. }\end{array}$ & 2008 & Paraná & $\begin{array}{l}\text { Arq Ciência e } \\
\text { Saúde UNIPAR }\end{array}$ & Lilacs \\
\hline $\begin{array}{l}\text { Beccaria LM et al. } \\
\text { [32] }\end{array}$ & $\begin{array}{l}\text { Eventos adversos na assistência de } \\
\text { enfermagem em unidade de terapia } \\
\text { intensiva. }\end{array}$ & 2009 & São Paulo & $\begin{array}{l}\text { Rev Bras Ter } \\
\text { Intensiva }\end{array}$ & Scielo \\
\hline $\begin{array}{l}\text { Gimenes FRE et al. } \\
\text { [33] }\end{array}$ & $\begin{array}{l}\text { Influência da redação da prescrição } \\
\text { médica na administração de medi- } \\
\text { camentos em horários diferentes do } \\
\text { prescrito. }\end{array}$ & 2009 & $\begin{array}{l}\text { São Paulo, } \\
\text { Fortaleza, Rio } \\
\text { Branco, Goiânia } \\
\text { e Campinas }\end{array}$ & $\begin{array}{l}\text { Acta Paul } \\
\text { Enferm }\end{array}$ & Scielo \\
\hline
\end{tabular}




\begin{tabular}{|l|l|l|l|l|l|}
\hline \multicolumn{1}{|c|}{ Autores } & \multicolumn{1}{|c|}{ Título do artigo } & Ano & Local do estudo & \multicolumn{1}{c|}{ Periódico } & \multicolumn{1}{c|}{$\begin{array}{c}\text { Base de } \\
\text { dados }\end{array}$} \\
\hline $\begin{array}{l}\text { Pelliciotti JSS et al. } \\
\text { [34] }\end{array}$ & $\begin{array}{l}\text { Erros de medicação e qualidade de } \\
\text { vida relacionada à saúde de profissio- } \\
\text { nais de enfermagem em unidades de } \\
\text { terapia intensiva. }\end{array}$ & 2010 & São Paulo & $\begin{array}{l}\text { Rev Latinoam } \\
\text { Enferm }\end{array}$ & Lilacs \\
\hline $\begin{array}{l}\text { Renovato RD et al. } \\
\text { [35] }\end{array}$ & $\begin{array}{l}\text { Investigação da técnica de adminis- } \\
\text { tração de medicamentos por sondas } \\
\text { enterais em hospital geral. }\end{array}$ & 2010 & $\begin{array}{l}\text { Dourado, Mato } \\
\text { Grosso do Sul }\end{array}$ & $\begin{array}{l}\text { Rev Enferm } \\
\text { UERJ }\end{array}$ & Lilacs \\
\hline $\begin{array}{l}\text { Santos JO et al. } \\
\text { [36] }\end{array}$ & $\begin{array}{l}\text { Condutas adotadas por técnicos de } \\
\text { enfermagem após ocorrência de erros } \\
\text { de medicação. }\end{array}$ & 2010 & Goiânia & $\begin{array}{l}\text { Acta Paul } \\
\text { Enferm }\end{array}$ & Lilacs \\
Scielo
\end{tabular}

\section{Caracterizaçáo dos erros encontrados e recomen- daçóes para a prática de enfermagem}

Outro aspecto a ser considerado é que as pesquisas apontaram os erros mais frequentes, e também relacionaram fatores potenciais para erros de medicação tais como: elevada carga horária de trabalho dos profissionais de enfermagem, formação insuficiente em farmacologia, problemas de comunicaçáo da equipe multiprofissional, dificuldade de interpretação da prescrição médica devido à ilegibilidade e uso de abreviaturas e medicamentos com nomes similares [14].

Segundo descrito em estudo [15], alguns fatores relatados por profissionais de enfermagem que podem desencadear em erros de medicação compreendem um grupo de causas associadas ao profissional (falta de atenção, falta de treinamento, falta de conhecimento, falta de comunicação, negligência e excesso de trabalho), e um segundo grupo associado ao sistema (ausência da dose unitária, problemas de prescriçáo médica e falhas gerais no sistema de medicação).

Pesquisa multicêntrica [40] identificou 74 erros de medicação ocorridos em hospitais brasileiros após a observação direta de 821 doses de medicamentos e os categorizou em: erro de dose
$24,3 \%$, erro de horário 22,9\%, medicamentos não autorizados $13,5 \%$ e erro de técnica 12,2\%. Pesquisa anterior de 2004 realizado no Estado de São Paulo evidenciou que $29 \%$ dos erros estavam relacionados à prescriçáo, 20,6\% ao horário e $13,6 \%$ ao preparo dos medicamentos [2]. Este contexto demonstra que os erros seguem as características do sistema de medicação próprio de cada instituição, e que pesquisas são necessárias nos diferentes cenários hospitalares de forma que medidas reparadoras sejam mais eficazmente propostas.

Os estudos analisados nesta revisão não demonstraram o impacto direto dos erros de medicação encontrados em relação ao agravamento do paciente, como, por exemplo, incapacidade física ou óbito.

Sobre as recomendaçóes para a prática da enfermagem, aponta-se que soluçóes específicas para a correção de erros de medicaçáo podem ser: padronização de medicamentos, dupla checagem, dose unitária, remoção de alguns medicamentos de áreas de fácil acesso, solucionar definitivamente o problema de embalagens e nomes semelhantes dos medicamentos e inclusão do farmacêutico clínico [12].

A implementação de prescriçóes informatizadas, uso de código de barras e de bombas de infusão inteligentes são estratégias capazes de reduzir os 
erros de medicaçáo relacionados à prática da enfermagem, pois envolvem suporte para as açóes de planejamento e se estendem até a implementação da terapia medicamentosa no paciente.

Pesquisa realizada há mais de uma década já sugeria recomendaçóes de acordo com categorias temáticas de erros, distribuídas da seguinte forma: falha no cumprimento de políticas e procedimentos - há a necessidade do cumprimento e revisão dos cinco "certos" no preparo e administração do medicamento, utilização da pulseira no antebraço, a adequação dos recursos humanos e carga de trabalho; falha no sistema de distribuição e preparo dos medicamentos pela farmácia - propóem-se novas formas de distribuição do medicamento, como a implantação do sistema de dose única; falha na comunicação - entre a equipe multiprofissional, recomendou-se a automatizaçáo do sistema para promover uma comunicação rápida, segura e atualizada sobre alteraçóes da prescrição médica e mudanças na terapia medicamentosa do paciente. Em relação à categoria falha no conhecimento, há evidências da necessidade de educação contínua e reciclagem profissional pelo enfermeiro e sua equipe, no que concerne à atualizaçáo de conhecimentos acerca da administração de medicamentos [16].

Foram recomendadas ações para melhorar o sistema de medicação e reduzir as oportunidades de erros na medicaçáo dividindo-as quanto à cronologia dos resultados esperados em: curto prazo - treinamentos periódicos sobre todas as fases do sistema e para todos os profissionais, criação de uma comissáo multidisciplinar envolvida com os aspectos da segurança de pacientes na prevençáo e reduçáo dos eventos adversos aos medicamentos, manual que contenha as interaçóes medicamentosas e as estabilidades dos medicamentos disponíveis para todos os profissionais em todas as salas de preparo, padronizar a maneira de prescrever os nomes dos medicamentos assim como a forma da dose sem uso de decimais; médio prazo - todos os médicos ingressos na residência devem passar por treinamento a respeito dos cuidados com a prescrição de medicamentos; longo prazo - implantação da prescrição por sistema computadorizado, implantação da dose unitária, utilização do código de barras na administração de medicamentos [23].

Também foi referida como sugestão para mudanças na prática de enfermagem a internalização da cultura de segurança do paciente, bem como a compreensão do erro de medicação como um processo multifatorial e sistêmico, deslocando desta forma o enfoque da culpa dos indivíduos a uma análise mais global do problema. Propóe-se ainda, criação e implementação de protocolos e formulários para notificação e monitoração de erros na administração de medicamentos, programas amplos dirigidos a toda a equipe de profissionais, ao local de trabalho e à instituiçáo como um todo, pois a reação e a atitude que se têm frente a ocorrência de um erro depende de como o mesmo é entendido [6,8,39].

Em conformidade com a tendência internacionalmente aceita, acredita-se que o incentivo para a notificação dos erros, a monitorização das ocorrências e fatores a elas relacionados, bem como açôes menos punitivas e mais educativas venham favorecer a diminuição dos erros que tantos malefícios causam aos pacientes e a todos os envolvidos na sua assistência, como ficou demonstrado nesta investigação.

\section{Conclusão}

Por meio desta revisão integrativa foi possível conhecer o estado da arte sobre erros de medicação com enfoque na enfermagem, ressaltando que a maioria dos artigos analisados deteve-se na determinação da incidência de erros, formas de notificação dos mesmos e as recomendaçóes para a prática de enfermagem, ficando esta lacuna ainda a ser preenchida.

Nenhum estudo evidenciou a vivência da experiência de um programa de redução dos erros de medicação em enfermagem. Sugere-se, portanto, realizaçáo de estudos que demonstrem o impacto direto dos erros de medicaçáo encontrados em relação ao agravamento do paciente, custos financeiros gerados a partir desses erros, e avaliaçáo de programas que sejam eficazes e efetivos na reduçáo do problema identificado.

\section{Referências}

1. NCCMERP, National Coordinating Council for Medication Error Reporting and Prevention. NCCMERP Taxonomy of Medication Errors. [citado 2011 Fev 2]. Disponível em URL:http://www.nccmerp.org/pdf/ taxo2001-07-31.pdf

2. Silva AEBC, Cassiani SHB. Erros de medicação em um hospital universitário: tipos, causas, sugestóes e providências. Rev Bras Enferm 2004;57(6):671-4.

3. Bates DW. Using information technology to reduce rates of medication errors in hospitals. BMJ 2000;320(7237):788-91.

4. Kohn LT, Corrignan JM, Donaldson MS. To err is human. Building a safer health system. Washington (DC): National Academy Press; 2000. 
5. World Health Organization. World Alliance for patient safety. [on-line] 2004 [citado 2011 Fev 2]. Disponível e http://www.who.int/patientsafety/en/brochure_final.pdf.

6. Cassiani SHB. A segurança do paciente e o paradoxo no uso de medicamentos. Rev Bras Enferm 2005;58(1):95-9.

7. Yamanaka TI, Pereira DG, Pedreira MLG, Peterlini MAS. Redesenho de atividades da enfermagem para redução dos erros de medicação em pediatria. Rev Bras Enferm 2007;60(2):190-6.

8. Silva AEB, Cassiani SHB, Miasso AI, Opitz SP. Problemas na comunicação: uma causa possível de erros de medicação. Acta Paul Enferm 2007;20(3):272-6.

9. Miasso AI, Silva AEBC, Cassiani SHB, Grou CR, Oliveira RC, Fakih FT. O processo de preparo e administração de medicamentos: identificação de problemas para propor melhorias e prevenir erros de medicação. Rev Latinoam Enferm 2006;14(3):354-63.

10. Souza MT, Silva MD, Carvalho R. Revisão integrativa: o que é e como fazer? Einstein 2010;8(1):102-6.

11. Therrien SMN, Almeida MI, Silva MGC. Ensino de enfermagem no Ceará de 1942-1956: a memória que projeta o futuro. Rev Bras Enferm 2008;16(1):125-30.

12. Wachter RM. Compreendendo a segurança do paciente. Porto Alegre: Artmed; 2010.

13. Polit DF, Beck CT, Hungler BP. Fundamentos de pesquisa em enfermagem: métodos, avaliação e utilização. $5^{\mathrm{a}}$ ed. Porto Alegre: Artmed; 2004.

14. Carvalho VT, Cassiani SHB. Erros na medicação: análise das situações relatadas pelos profissionais de enfermagem. Medicina 2000;33:322-30.

15. Carvalho VT, Cassiani SHB. Análise dos comportamentos dos profissionais de enfermagem frente aos erros na administraçáo de medicamentos. Acta Paul Enferm 2002;15(2):45-54.

16. Camargo MNV, Padilha KG. Ocorrências iatrogênicas com medicação em unidades de terapia intensiva. Acta Paul Enferm 2003;16(4):69-76.

17. Carvalho VT, Cassiani SHB. Erros na medicação e consequências para profissionais de enfermagem e clientes: um estudo exploratório. Rev Latinoam Enferm 2002;10(4):523-9.

18. Bohomol E, Ramos LH. Erros de medicação: causas e fatores desencadeantes sob a ótica da equipe de enfermagem. Acta Paul Enferm 2003;16(2):41-8.

19. Padilha KG, Kitahara PH, Gonçalves CCS, Sanches ALC. Ocorrências iatrogênicas com medicação em unidade de terapia intensiva: condutas adotadas e sentimentos expressos pelos enfermeiros. Rev Esc Enferm USP 2002;36(1):50-7.

20. Cassiani SHB, Freire CC, Gimenes FRE. A prescrição médica eletrônica em um hospital universitário: falhas de redação e opiniōes de usuários. Rev Esc Enferm USP 2003;37(4):51-60.

21. Santos AE, Padilha KG. Eventos adversos com medicação em Serviços de Emergência: condutas profissionais e sentimentos vivenciados por enfermeiros. Rev Bras Enferm 2005;58(4):429-33.

22. Melo LR, Pedreira MLG. Erros de medicação em pediatria: análise da documentação de enfermagem no prontuário do paciente. Rev Bras Enferm 2005;58(2):180-5.

23. Oliveira RC, Camargo AEB, Cassiani SHB. Estratégias para prevençáo de erros de medicação no setor de emergência. Rev Bras Enferm 2005;58(4):399-404.
24. Cassiani SHB, Teixeira TCA, Opitz SP, Linhares JC. O sistema de medicação nos hospitais e sua avaliação por um grupo de profissionais. Rev Esc Enferm USP 2005;39(3):280-7.

25. Toffoletto MC, Padilha KG. Consequências de erros de medicaçáo em unidades de terapia intensiva e semi-intensiva. Rev Esc Enferm USP 2006;40(2):247-52.

26. Bohomol E, Ramos LH. Perceptions about medication errors: analysis of answers by the nursing team. Rev Latinoam Enferm 2006;14(6):887-92.

27. Santos JO, Silva AEBC, Munari DB, Miasso AI. Sentimentos de profissionais de enfermagem após a ocorrência de erros de medicação. Acta Paul Enferm 2007;20(4): 483-8.

28. Bohomol E, Ramos LH. Erro de medicação: importância da notificaçáo no gerenciamento da segurança do paciente. Rev Bras Enferm 2007;60(1):32-6.

29. Silva DO, Grou CR, Miasso AI, Cassiani SHB. Medication preparation and administration: analysis of inquiries and information by the nursing team. Rev Latinoam Enferm 2007;15(5):1010-7.

30. Silva AEBC, Cassiani SHB, Miasso AI, Optiz SP. Problemas na comunicação: uma possível causa de erros de medicação. Acta Paul Enferm 2007;20(3):272-6.

31. Freitas DF, Yasuo OJ. Avaliação dos fatores de risco relacionados às falhas durante a administraçáo de medicamentos. Arq Ciênc Saúde UNIPAR 2008;12(3):231-7.

32. Beccaria LM, Pereira RAM, Contrin LM, Lobo A,Trajano DHL. Eventos adversos na assistência de enfermagem em uma unidade de terapia intensiva. Rev Bras Ter Intensiva 2009;21(3):276-8.

33. Gimenes FRE, Teixeira TCA, Silva AEBC, Optiz SP, Mota MLS, Cassiani SHB. Influência da redação da prescrição médica na administração de medicamentos em horários diferentes do prescrito. Acta Paul Enferm 2009;22(4):380-4.

34. Pelliciotti JSS, Kimura M. Erros de medicação e qualidade de vida relacionada à saúde de profissionais de enfermagem em unidades de terapia intensiva. Rev Latinoam Enferm 2010; 18(6):1062-69.

35. Renovato RD, Carvalho PD, Rocha RSA. Investigação da técnica de administração de medicamentos por sondas enterais em um Hospital Geral. Rev Enferm UERJ 2010; 18(2):173-8.

36. Santos JO, Silva AEBC, Munari DB, Miasso AI. Condutas adotadas por técnicos de enfermagem após ocorrência de erros de medicação. Acta Paul Enferm 2010;23(3):328-33.

37. Franco JN, Ribeiro G, D'Innocenzo M, Barros, Amaral BP. Percepçáo da equipe de enfermagem sobre fatores causais de erros na administração de medicamentos. Rev Bras Enferm 2010;63(6):927-32.

38. Belela ASC, Peterlini MAS, Pedreira MLG. Revelação da ocorrência de erro de medicação em unidade de cuidados intensivos pediátricos. Rev Bras Ter Intensiva 2010;22(3):257-63.

39. Pereira CMB, Pereira OB, Carboni RM. Conduta do futuro enfermeiro mediante o erro de medicação. Rev Enferm UFPE 2010;4(1):113-8.

40. Gimenes FREG, Mota MLS, Teixeira TCA, Silva AEBC, Opitz SP, Cassiani SHB. Patient safety in drug therapy and the influence of the prescription in dose errors. Rev Latinoam Enferm 2010;18(6):1055-61. 